BNL-52348

AGS/AD/93-1

UC-414 (High Energy PhysicsDOE/OSTI-4500-RZ5)

BNL -52348

DE93 004633

\title{
RADIATION FROM MOVING CHARGED \\ PARTICLES WITH SPIN
}

\author{
Alfredo Luccio
}

October 5, 1992

\section{ALTERNATING GRADIENT SYNCHROTRON DEPARTMENT \\ BROOKHAVEN NATIONAL LABORATORY ASSOCIATED UNIVERSITIES, INC. UPTON, LONG ISLAND, NEW YORK}

UNDER CONTRACT NO. DE-AC02-76CH00016 WITH THE UNITED STATES DEPARTMENT OF ENERGY 


\title{
RADIATION FROM MOVING CHARGED PARTICLES WITH SPIN
}

\author{
Alfredo Luccio
}

\begin{abstract}
The theory of radiation emitted by a charged particle with spin in relativistic motion in an external magnetic field is reviewed. Approximate expressions suitable for numerical computation, in far and near field, are derived. In particular, the case of the passage of a particle accelerator beam through an undulator is considered. It is shown that observation of the spectrum of the emitted radiation, in its two states of polarization, can be used not only for beam diagnostics, but also to measure the spin state of the accelerated particles. Undulator radiation is compared with Compton scattering of laser light by the particle beam. Examples for high energy electron and proton colliders are presented.
\end{abstract}




\section{Introduction}

Particles in the beam of a storage ring or collider may have a high degree of spin polarization. Siberian snakes and spin rotators are being used to preserve and control the direction of the spin for experiments in Nuclear Physics ${ }^{1}$.

Rotation of the spin can be achieved by an arrangement of magnets producing fields at right angle with the direction of propagation of the beam. In the design of a spin rotator, a first condition is that a particle entering the device on axis and with zero angle with it, emerges again on axis and at zero angle, which is obtained by designing dipole arrays with overall first and second field integrals as close to zero as possible ${ }^{2}$. Another condition is that in the passage through the rotator the deviation of the particle trajector. $\mathrm{sm}$ the accelerator equilibrium orbit and the trajectory angle should be kept as small as possible, in order to avoid the unavoidable field distortions that take place at locations far from the equilibrium orbit. This latter condition is particularly important in accelerators where the final energy is much larger than the injection energy, thus producing a large range of excursions for the orbit in the transverse rotator.

To achieve a given spin rotation, a given value of the field integral in each dipole is required. In principle, it is equivalent to build a rotator with few long dipoles or with many short diroles. On the other hand, the maximum orbit deviation in a magnet dipole array is proportional to the length of the field of a single dipole, so it appears convenient to build a rotator with many dipoles. We have proposed to build spin rotators with two transverse wigglers of many poles with fields perpendicular to each other, longitudinally shifted ${ }^{3}$. We have shown that this arrangement allows one to achieve a given spin rotation with a lesser degree of orbit deformation, using much smaller magnets and lower electrical power than in more established structures.

An added advantage of wiggler spin rotators is that they can be also used as beam diagnostic tools, allowing measurements of the beam size and spin polarization from observation of the synchrotron radiation spectrum, a highly accuraie and non destructive methodology. Synchrotron radiation photon flux is not only conveniently measured for electrons, but also for high energy protons in modern storage rings and colliders ${ }^{4}$. Beam diagnostics can be done with the SR spectral continuum from bending magnets, however the spectrum of wigglers being the result of interference presents a characteristic line structure ai.d therefore has a higher specific brightness. With SR one can produce an image of the beam that allows one to measure beam size and position. Spectral line frequency, shape and polarization contains other information on the source beam, including particle energy and spin state. In conclusion, if the concept of a multi period spin rotator appears viable, the very same device may provide beam diagnostics for free. 
At present, spin polarization is being measured by Compton backscattering of laser light by the particle beam. The physical mechanism of photon production by wiggler radiation and backscattering is the same, and. lead to comparable results. However, laser backscattering produces a continuum of photon energies, as the SR from a bending magnet does, (even if in the former case the energy is correlated with the scattering angle:), and many of the nice properties of the enhanced wiggler spectrum are lost.

In this paper the relevant formalism of the radiation of a particle with spin in relativistic motion through the field of a wiggler is reviewed. The results are then compared with Compton backscattering. In particular, the ratio of the yield of the spin-dependent part of the spectrum to the spin-independent part is estimated, and finally the results are applied to high energy electron and proton storage rings: NSLS-X, APS-Argonne, RHIC (protons), SSC.

We use MKSA units throughout this paper. Main reference is Jackson's ${ }^{5}$.

\section{Poynting vector}

The starting point to the theory of the radiation of a relativistic particle moving through a magnetic field is to examine the Poynting vector of the electromagnetic field. A particle of mass $m$ and charge $e$ is accelerated in a magnetic field $\mathbf{B}$. The frame of reference is shown in figure 1 . The preferred direction of motion is along $z(\mathbf{k})$. The horizontal plane is $x-z$, the radial direction is $x(\mathbf{i})$, the vertical direction is $y(\mathbf{j})$.

In a given point of space the radiated power flux is given by the Poynting theorem

$$
\frac{d \mathbf{P}}{d \sigma}\left[\text { watt } / \mathrm{m}^{2}\right]=\mathbf{E} \times \mathbf{B} \quad ; \quad \frac{d \mathbf{P}}{d \Omega}[\text { watt } / \text { sterad }]=\frac{R^{2} E^{2}}{\mu_{0} c}
$$

with $R$ the distance between the charge and the field point. If we only retain the "radiation" term in the expression for the field $\mathbf{E}$ (i.e. the term $\propto 1 / R$ ), it is

$$
R E=\frac{\mu_{0}}{4 \pi} e c|\mathbf{A}(t)| ; 4 \pi \frac{d P}{d \Omega}[\text { watt } / \text { sterad }]=\frac{\mu_{0}}{4 \pi} e^{2} c|\mathbf{A}(t)|^{2}
$$

The vector potential $\mathbf{A}=\mathbf{A}(t)$ is

$$
\mathbf{A}=\frac{\mathbf{n} \times\left[(\mathbf{n}-\beta) \times \frac{d \beta}{d t}\right]}{[1-\mathbf{n} \cdot \beta]^{3}}
$$




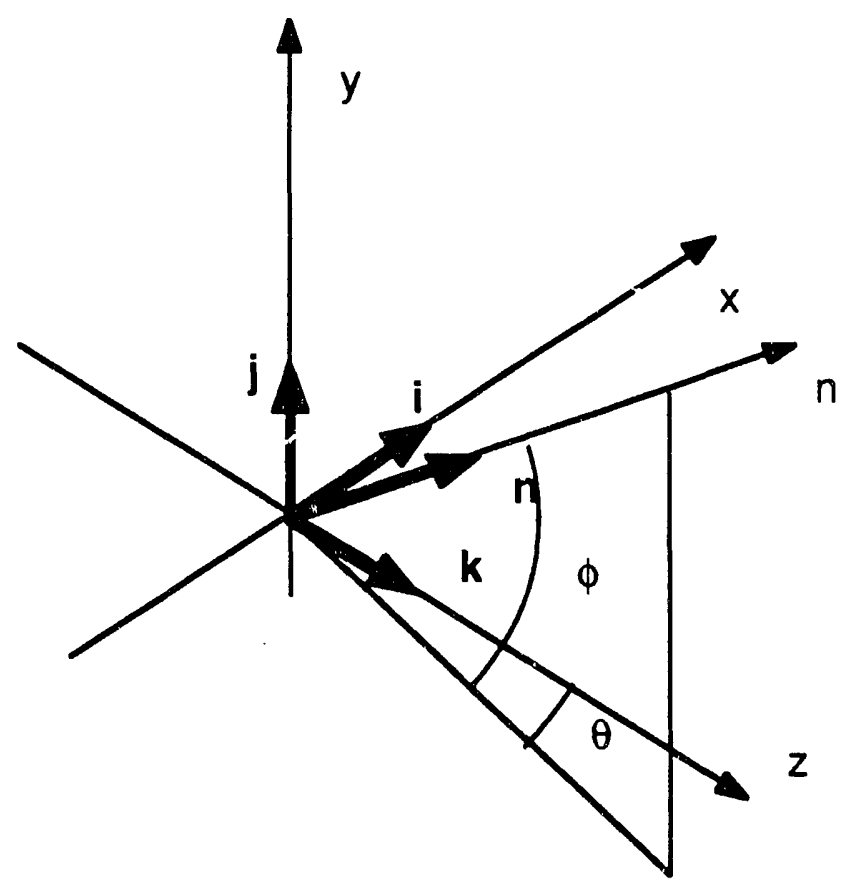

Fig. 1. The unit vector $\mathbf{n}$ points towards the observation point.

The total energy radiated by the particle in a time interval $\Delta t$ is

$$
4 \pi \frac{d W}{d \Omega}[\text { joule } / \text { sterad }]=\frac{\mu_{0}}{4 \pi} e^{2} c \int_{\Delta t}|\mathbf{A}(t)|^{2} d t
$$

To calculate the spectrum, the vector potential will be sampled at equal intervals in the retarded time $t_{R}$ (i.e. the time at the observation point) and then a Fourier transform will be performed. Replace $t$ with $t R$ in the integral of Eq. (4) and apply Parseval's Theorem

$$
\int\left|\mathbf{A}\left(t_{R}\right)\right|^{2} d t_{R}=\int|\mathbf{A}(\omega)|^{2} d \omega
$$

Then, using the definition of the energy of a photon of frequency $\omega$ and the MKSA definition of the fine structure constant $\alpha$

$$
\varepsilon=\hbar \omega \quad ; \quad \alpha=" \frac{1}{137} "=\frac{\mu_{0}}{4 \pi} \frac{e^{2} c}{\hbar}
$$

and introducing the source current $I$ [amp], obtain for the number of photons emitted per second, per unit solid angle and per unit photon energy interval

$$
\frac{d^{2} n}{d \Omega d \varepsilon}[\text { photons } /(\text { sec - sterad - joule })]=\frac{\alpha I}{2 \pi e}|\mathbf{A}(\omega)|^{2}
$$




\section{Equation of the trajectory}

If we assume that the energy of the particle remains constant, i.e. that the energy radiated away is negligible, in Eq.(3), the particle velocity $\beta$ and its time derivative in the magnetic field are calculated from the equation of motion

$$
\frac{d \beta}{d t}=\beta \times \Omega ; \Omega=\frac{e \mathbf{B}}{m \gamma}
$$

Explicitly, for the components of $\beta$ we have

$$
\begin{aligned}
\frac{d}{d z} \beta_{x} & =\frac{1}{\gamma c \beta_{z}}\left(\beta_{y} \Omega_{z}-\beta_{z} \Omega_{y}\right) \\
\frac{d}{d z} \beta_{y} & =\frac{1}{\gamma c \beta_{z}}\left(\beta_{z} \Omega_{x}-\beta_{x} \Omega_{z}\right) \\
\beta_{z}^{2} & =\beta^{2}-\left(\beta_{x}^{2}+\beta_{y}^{2}\right)
\end{aligned}
$$

The equations for the particle trajectory and angles are

$$
\begin{aligned}
& \frac{d x}{d z}=\frac{\beta_{x}}{\beta_{z}}=\theta_{b} \\
& \frac{d y}{d z}=\frac{\beta_{y}}{\beta_{z}}=\phi_{b}
\end{aligned}
$$

\section{Far field formulation}

In far field (Fraunhofer), the radiation will be calculated along the direction defined by the unitary' vector $\mathbf{n}$, with angles $\theta$ and $\phi$. Vector potential components $A_{\mathrm{p}}$, parallel to the horizontal plane and perpendicular to $\mathbf{n}$, and $A_{\sigma}$, perpendicular to $\mathbf{n}$ and to $A_{\mathrm{p}}$ are

$$
A_{\rho}=\mathbf{A} \cdot[\mathbf{j} \times \mathbf{n}] ; A_{\sigma}=\mathbf{A} \cdot\{[\mathbf{j} \times \mathbf{n}] \times \mathbf{n}\}
$$

After some vector algebra, making use of the vector identities

$$
\mathbf{a} \times \mathbf{b} \cdot \mathbf{c}=\mathbf{b} \times \mathbf{c} \cdot \mathbf{a}=\mathbf{c} \times \mathbf{a} \cdot \mathbf{b} \quad ; \quad \mathbf{a} \times[\mathbf{b} \times \mathbf{c}]=[\mathbf{c} \cdot \mathbf{a}] \mathbf{b}-[\mathbf{b} \cdot \mathbf{a}] \mathbf{c}
$$

the explicit expressions are found

$$
A_{p}=\frac{1}{D^{3}} \mathbf{P} \cdot \Omega \quad ; \quad A_{\sigma}=\frac{1}{D^{3}} \mathbf{S} \cdot \Omega
$$


with

$$
\begin{gathered}
D=1-\beta \cdot \mathbf{n} \\
\mathbf{P}=\left(\begin{array}{c}
-n_{y} \beta_{x}(\beta \cdot \mathbf{n})+n_{x} n_{y} \beta^{2}+\beta_{y}\left(\beta_{x}-n_{x}\right) \\
\left(1-n_{y} \beta_{y}\right)(\beta \cdot \mathbf{n})+\left(n_{y}^{2}-1\right) \beta^{2}+\beta_{y}\left(\beta_{y}-n_{y}\right) \\
-n_{y} \beta_{z}(\beta \cdot \mathbf{n})+n_{z} n_{y} \beta^{2}+\beta_{y}\left(\beta_{z}-n_{z}\right)
\end{array}\right) \\
\mathbf{S}=\left(\begin{array}{c}
\left(n_{x}-\beta_{x}\right)(\beta \times \mathbf{n})_{y}-n_{z}\left(\beta^{2}-\beta \cdot \mathbf{n}\right) \\
\left(n_{y}-\beta_{y}\right)(\beta \times \mathbf{n})_{y} \\
\left(n_{z}-\beta_{z}\right)(\beta \times \mathbf{n})_{y}+n_{x}\left(\beta^{2}-\beta \cdot \mathbf{n}\right)
\end{array}\right) \\
\mathbf{n}=\left(\begin{array}{c}
\sin \theta \cos \phi \\
\sin \phi \\
\cos \theta \cos \phi
\end{array}\right) \\
\left\{\begin{array}{c}
\beta^{2}=1-\frac{1}{\gamma^{2}} \\
\beta \cdot \mathbf{n}=\beta_{x} n_{x}+\beta_{y} n_{y}+\beta_{z} n_{z} \\
(\beta \times \mathbf{n})_{y}=\beta_{z} n_{x}-\beta_{x} n_{z}
\end{array}\right.
\end{gathered}
$$

\section{Approximations}

To calculate the spectrum, the vector potential is calculated at equal intervals in the retarded time. In far field, the transformations between retarded time $t_{R}$, time at the trajectory and longitudinal coordinate are

$$
\frac{d t_{R}}{d t}=1-\beta \cdot \mathbf{n} ; \frac{d z}{d t}=\beta_{z} c
$$

When the beta factors are very close to one, the errors made in the numerical integration of the equations of motion and in the calculation of the vector potential may be large. This problem is solved expanding all the quantities in power of the transverse angles of motion. To second order in $\theta, \phi, \theta_{\mathrm{b}}, \phi_{\mathrm{b}}$ and $1 / \gamma_{\mathrm{c}}$, obtain

$$
\mathbf{P} \cong \frac{1}{2 \gamma^{2}}\left(\begin{array}{c}
2 \gamma^{2} \Delta \theta \Delta \phi \\
1-\gamma^{2}\left[\Delta \theta^{2}-\Delta \phi^{2}\right] \\
-\phi_{b}+\gamma^{2}\left[\phi \Delta \theta^{2}-\left(\theta+\theta_{b}\right) \Delta \theta \Delta \phi-\phi_{b} \Delta \phi^{2}\right]
\end{array}\right)
$$




$$
\mathbf{S} \cong \frac{1}{2 \gamma^{2}}\left(\begin{array}{c}
1+\gamma^{2}\left(\Delta \theta^{2}-\Delta \phi^{2}\right) \\
2 \gamma^{2} \Delta \theta \Delta \phi \\
-\theta_{b}-\gamma^{2}\left[\theta_{b} \Delta \theta^{2}+\left(\phi+\phi_{b}\right) \Delta \theta \Delta \phi-\theta \Delta \phi^{2}\right]
\end{array}\right)
$$

where

$$
\Delta \theta=\theta-\theta_{b} ; \Delta \phi=\phi-\phi_{b}
$$

The equations of motion, using as variables the beam angles, become

$$
\begin{aligned}
& \frac{d \theta_{b}}{d z}=\frac{1}{\gamma \beta_{z} c}\left[\theta_{b} \phi_{b} \Omega_{x}-\left(1+\theta_{b}^{2}\right) \Omega_{y}+\phi_{b} \Omega_{z}\right] \\
& \frac{d \phi_{b}}{d z}=\frac{1}{\gamma \beta_{z} c}\left[\left(1+\phi_{b}^{2}\right) \Omega_{x}-\theta_{b} \phi_{b} \Omega_{y}-\theta_{b} \Omega_{z}\right] \\
& \beta_{z}=1-\frac{1}{2 \gamma^{2}}\left[1+\gamma^{2}\left(\theta_{b}^{2}+\phi_{b}^{2}\right)\right]
\end{aligned}
$$

and the transformation between retarded time and particle time

$$
\frac{d t_{R}}{d t}=D \cong \frac{1}{2 \gamma^{2}}\left\{1+\gamma^{2}\left[\Delta \theta^{2}+\Delta \phi^{2}\right]\right\}
$$

\section{Near field.}

In near field (Fresnel, figure 2), when the distance $R$ of the observation point $\mathbf{P}$ from the radiating particle $\mathbf{Q}$ is not too large in comparison with the distance traveled by the particle in the time interval $\Delta t$, the radiation should be calculated in a different way.

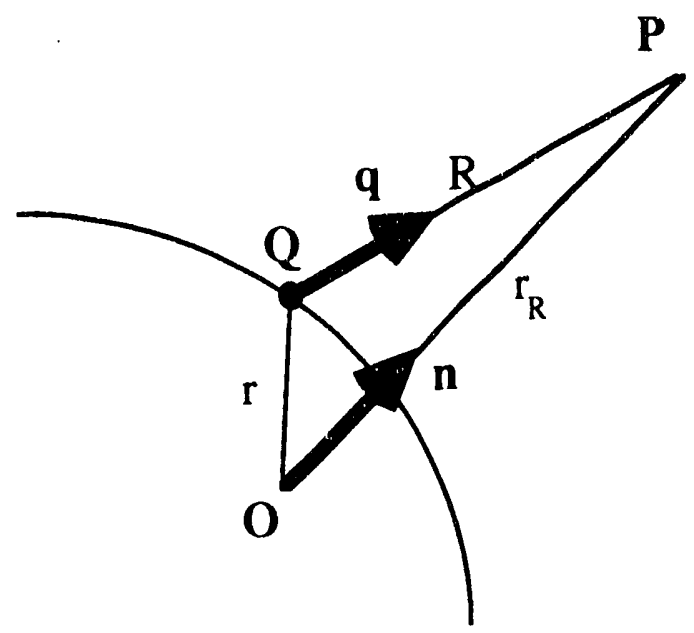

Fig.2. Near field. $Q$ is the radiating particle, $P$ the observation point. 
The variation of $R$ itself with time is not a factor since it is the product $R E$ that enters the expression for the power spectrum, however the transformation (19) between the time $t$ at the particle and the retarded time $t_{R}$ at the observation point has a different expression, namely

$$
\frac{d t_{R}}{d t}=1-\beta \cdot \hat{\mathbf{q}} \quad ; \quad \hat{\mathbf{q}}=\frac{\mathbf{r}_{R}-\mathbf{r}}{R}
$$

Also the meaningful components of the radiation probably are the ones lying on the observation plane (perpendicular to $\mathbf{k}$, the $z$-axis, figure 3), parallel and perpendicular to the horizontal plane, respectively

$$
A_{x}=\mathbf{A} \cdot \mathbf{i}=A_{p} \cos \theta ; A_{y}=\mathbf{A} \cdot \mathbf{j}=A_{\sigma} \cos \phi
$$

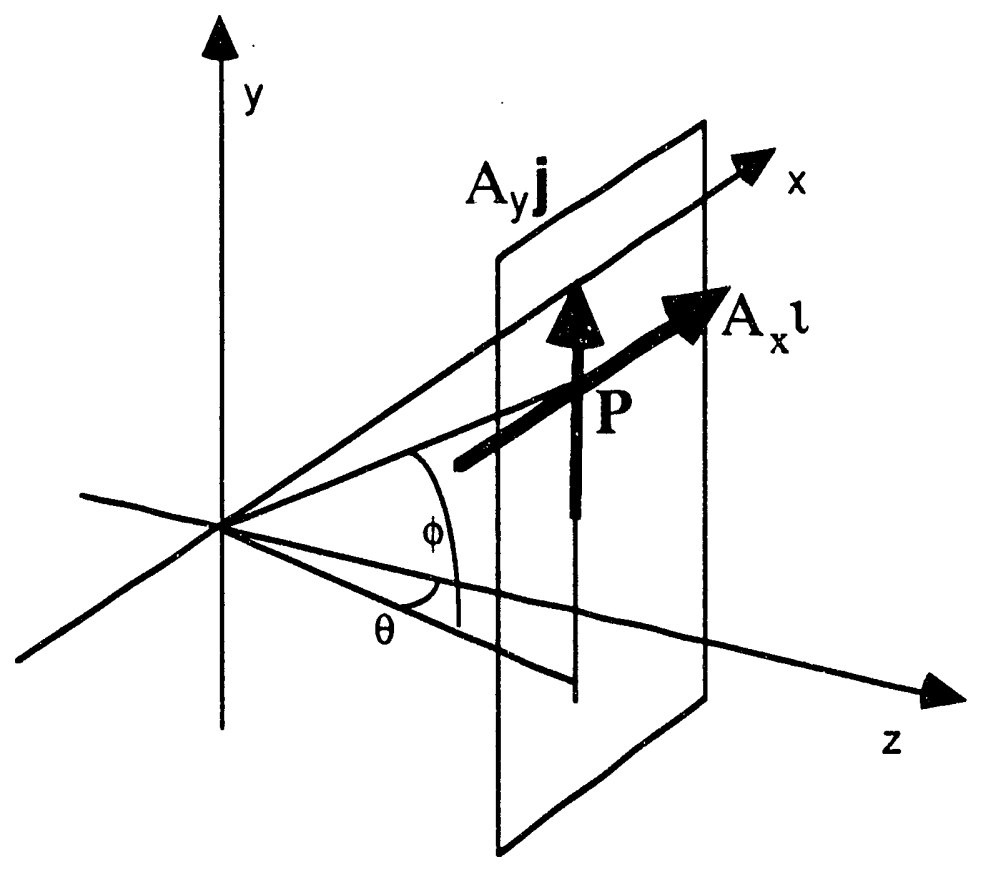

Fig.3. Geometry for near field.

To calculate far field radiation, only the instantaneous velocity $\beta$ of the particle is needed, and then the equation of motion (8) has to be integrated only once. To calculate near field radiation, because of the different time transformation, also the trajectory, $\mathbf{r}(t)$ should be known. This implies a further integration.

\section{Effect of the particle spin}

The magnetic moment $\mu$ of the charged part cle is proportional to the spin s (in units $\pm 1 / 2$ )

$$
\mu=\frac{\varepsilon e}{m c} \frac{\hbar}{2} s
$$


$\mathbf{S}$ is the particle spin in the particle rest frame (PRF) In the LAB frame, the spin transforms as follows

$$
\mathbf{S}=\mathbf{s}+\frac{\gamma^{2}}{\gamma+1}(\beta \cdot \mathbf{s}) \beta
$$

The particle spin, in its precession in an external magnetic field, adds to the radiation field of Eq. (3) a vector potential contribution

$$
\mathbf{A}^{(s)}(t)=\frac{1}{(1-\boldsymbol{\beta} \cdot \mathbf{n})} \frac{d}{d t}\left[\frac{\mathbf{n} \times[\mathbf{S}+\mathbf{n} \times(\boldsymbol{\beta} \times \mathbf{S})]}{(1-\beta \cdot \mathbf{n})}\right]
$$

Using the explicit expression in Eq. (27), the following is obtained

$$
\mathbf{A}^{(s)}(t)=\frac{\vdots}{(1-\beta \cdot \mathbf{n})} \frac{d}{d t}\left[\frac{\mathbf{n} \times[\mathbf{s}+\mathbf{n} \times(\beta \times \mathbf{s})]}{(1-\beta \cdot \mathbf{n})}+\frac{\gamma^{2}}{\gamma+1} \frac{(\beta \cdot \mathbf{s}) \mathbf{n} \times \boldsymbol{\beta}}{(1-\beta \cdot \mathbf{n})}\right]
$$

In the cases we are interes:ed in, namely for $\gamma \gg>1$, the second term in the r.h.s. of Eq. (29) is much larger than the first, due to relativistic dilation. From now on, we will only consider this term. The total radiation field will be

$$
\mathbf{A}^{(T)}(t)=\mathbf{A}(t)+\eta \mathbf{A}^{(s)}(t)
$$

with the first term, the one given by Eq. (3) and the second term, given by Eq. (29), and with

$$
\eta=g \frac{\gamma^{2}}{\gamma+1} \frac{\hbar \omega}{4 m c^{2}} \cong \frac{g \gamma}{4} \frac{\hbar \omega}{m c^{2}}
$$

Eq. (31) shows that at a given photon energy the contribution of the spin to the radiation density is inversely proportional to the mass of the particle.

If we perform the time derivative in Eq. (29), find the following explicit expression for the spin dependent part of the radiation vector potential

$$
\begin{aligned}
\mathbf{A}^{\left(s^{\prime}\right.}(t)= & \frac{(1-\boldsymbol{\beta} \cdot \mathbf{n})[(\dot{\beta} \cdot \mathbf{s}) \mathbf{n} \times \boldsymbol{\beta}+(\boldsymbol{\beta} \cdot \mathbf{s}) \mathbf{n} \times \dot{\beta}]+(\boldsymbol{\beta} \cdot \mathbf{s})(\dot{\beta} \cdot \mathbf{n}) \mathbf{n} \times \boldsymbol{\beta}}{(1-\beta \cdot \mathbf{n})^{3}} \\
& +\frac{(1-\beta \cdot \mathbf{n})(\beta \cdot \dot{\mathbf{s}})}{(1-\beta \cdot \mathbf{n})^{3}} \mathbf{n} \times \beta
\end{aligned}
$$


where the first term contains the spin and the second its time derivative.

The spin s precedes in an external magnetic field, obeying to the BMT equation

$$
\frac{d \mathbf{s}}{d t}=C_{1} \mathbf{s} \times \Omega+C_{2}(\beta \cdot \Omega)(\mathbf{s} \times \beta)
$$

with

$$
C_{1}=1+G \gamma \quad ; \quad C_{2}=-\frac{G \gamma^{2}}{1+\gamma} ; G=g-2
$$

Since in Eq. (32) the time derivative of the spin appears in a scalar product with $\beta$, and in the BMT equation the spin appears in a cross product with $\beta$, then when we combine Eqs. (32) and (33), no term containing the coefficient $C_{2}$ will appear in the final expression.

After performing some vector algebra, the total vector potential can be written as follows (the nonspin dependent part has been rewritten for convenience)

$$
\begin{aligned}
& A_{p}^{(T)}(t)=A_{p}(t)+\eta A_{p}^{(s)}(t)=\frac{1}{2 \gamma^{2} D^{3}} \sum_{i}\left[p_{i}+\eta \sum_{j} p_{i j}^{(s)} s_{j}\right] \Omega_{i} \\
& A_{\sigma}^{(T)}(t)=A_{\sigma}(t)+\eta A_{\sigma}^{(s)}(t)=\frac{1}{2 \gamma^{2} D^{3}} \sum_{i}\left[\sigma_{i}+\eta \sum_{j} \sigma_{i j}^{(s)} s_{j}\right] \Omega_{i}
\end{aligned}
$$

(the indices $i, j=1,2,3$ mean " $x, y, z$ "), with the vectors $\mathbf{p}$ and $\sigma$ coincident with $\mathbf{P}$ and $\mathbf{S}$ of Eqs. (15) and (16), respectively, and with exact expressions for the tensors $p^{s_{i j}}$, $\sigma_{i j}$ given by

$$
\begin{aligned}
p_{i j}^{(s)} & =\left(\begin{array}{ccc}
\beta_{1} u_{1} & \beta_{1} u_{2}-a \beta_{3} & \beta_{1} u_{3}+a \beta_{2} \\
\beta_{2} u_{1}+a \beta_{3} & \beta_{2} u_{2} & \beta_{2} u_{3}-a \beta_{1} \\
\beta_{3} u_{1}-a \beta_{2} & \beta_{3} u_{2}+a \beta_{1} & \beta_{3} u_{3}
\end{array}\right) \\
\sigma_{i j}^{(s)} & =\left(\begin{array}{ccc}
\beta_{1} v_{1} & \beta_{1} v_{2}-b \beta_{3} & \beta_{1} v_{3}+b \beta_{2} \\
\beta_{2} v_{1}+b \beta_{3} & \beta_{2} v_{2} & \beta_{2} v_{3}-b \beta_{1} \\
\beta_{3} v_{1}-b \beta_{2} & \beta_{3} v_{2}+b \beta_{1} & \beta_{3} v_{3}
\end{array}\right)
\end{aligned}
$$

with the definitions

$$
\begin{aligned}
& \left\{\begin{array}{l}
a=\left(1-C_{1}\right)(1-\beta \cdot \mathbf{n})(\mathbf{m} \times \mathbf{n} \cdot \beta) ; \mathbf{m}=\mathbf{j} \times \mathbf{n} \\
\mathbf{u}=[a \mathbf{n}-(1-\beta \cdot \mathbf{n}) \mathbf{m} \times \mathbf{n}] \times \beta
\end{array}\right. \\
& \left\{\begin{array}{l}
b=\left(1-C_{1}\right)(1-\beta \cdot \mathbf{n})(\mathbf{l} \times \mathbf{n} \cdot \beta) ; \quad \mathbf{l}=\mathbf{j} \times \mathbf{n} \\
\mathbf{v}=[a \mathbf{n}-(1-\beta \cdot \mathbf{n}) \mathbf{l} \times \mathbf{n}] \times \boldsymbol{\beta}
\end{array}\right.
\end{aligned}
$$


To the lowest order in the beam and radiation angles and in the (inverse) energy, approximate expressions for the tensors $p$ and $\sigma$ are

$$
\begin{gathered}
\mathbf{p}=\left(\begin{array}{lll}
A & B & K
\end{array}\right) \\
\mathbf{p}^{(s)}=F\left(\begin{array}{ccc}
\theta_{b} & -\left(1-C_{1}\right) \Delta \phi & 0 \\
\phi-C_{1} \Delta \phi & 0 & 0 \\
1 & 0 & -\theta_{b}
\end{array}\right) \\
\sigma=\left(\begin{array}{lll}
H & A & M
\end{array}\right) \\
\sigma^{(s)}=F\left(\begin{array}{ccc}
0 & \theta-C_{1} \Delta \theta & 0 \\
\left(1-C_{1}\right) \Delta \theta & -\phi_{b} & 0 \\
0 & -1 & \phi_{b}
\end{array}\right)
\end{gathered}
$$

with $C_{I}$ the coefficient appearing in the BMT essation, and

$$
\begin{aligned}
& A=2 \gamma^{2} \Delta \theta \Delta \phi, \quad F=1+\gamma^{2}\left(\Delta \theta^{2}+\Delta \phi^{2}\right), \\
& B=1-\gamma^{2}\left(\Delta \theta^{2}-\Delta \phi^{2}\right), \quad H=1+\gamma^{2}\left(\Delta \theta^{2}-\Delta \phi^{2}\right), \\
& K=-\phi_{b}+\gamma^{2}\left(\phi \Delta \theta^{2}-\left(\theta+\theta_{b}\right) \Delta \theta \Delta \phi-\phi_{b} \Delta \phi^{2}\right), \\
& M=-\theta_{b}-\gamma^{2}\left(\theta_{b} \Delta \theta^{2}+\left(\theta+\theta_{b}\right) \Delta \theta \Delta \phi-\theta \Delta \phi^{2}\right)
\end{aligned}
$$

In the case of practical importance where only a transverse magnetic field is present, the third element in the vectors (39) and (41) and the third 'ine in the matrices (40) and (42) can be dropped. It is convenient to rewrite the equations (35) in the transverse field case and to lowest order in the angles and $1 / \gamma$

$$
\begin{aligned}
& A_{p}^{(T)}(t)=\frac{1}{2 \gamma^{2} D^{3}}\left\{\left[A-\eta F\left(\theta_{b} s_{x}+\left(\phi-C_{1} \Delta \phi\right) s_{y}+s_{z}\right)\right] \Omega_{x}+\left[B-\eta F\left(1-C_{1}\right) \Delta \phi s_{x}\right] \Omega_{y}\right\} \\
& A_{\sigma}^{(T)}(t)=\frac{1}{2 \gamma^{2} D^{3}}\left\{\left[H-\eta F\left(1-C_{1}\right) \Delta \theta s_{y}\right] \Omega_{x}+\left[A-\eta F\left(\left(\theta-C_{1} \Delta \theta\right) s_{x}+\phi_{b} s_{y}+s_{z}\right)\right] \Omega_{y}\right\}
\end{aligned}
$$




\section{On axis}

If the particle beam has no emittance, in a transverse field and for observation on axis, it is either $\Delta \phi=0$ or $\Delta \theta=0$. In both cases $A=0$.

In particular, if only $\Omega_{y} \neq 0$, then on axis $\Delta \phi=0$

$$
\begin{aligned}
& A_{p}=B \Omega_{y} \\
& A_{\sigma}=0 \\
& A_{p}^{(s)}=0 \\
& A_{\sigma}^{(s)}=-F\left[C_{1} \theta_{b} s_{x}+s_{z}\right] \Omega_{y}
\end{aligned}
$$

and, if only $\Omega_{\mathrm{x}} \neq 0$, then on axis $\Delta \theta=0$

$$
\begin{aligned}
& A_{p}=0 \\
& A_{\sigma}=H \Omega_{y} \\
& A_{p}^{(s)}=F\left[C_{1} \phi_{b} s_{x}+s_{z}\right] \Omega_{x} \\
& A_{\sigma}^{(s)}=0
\end{aligned}
$$

A first result is that a spin effect on the radiation pattern can be observed, on axis, in the spectrum polarized in a direction parallel to the magnetic field.

Let us look at the frequency spectrum of the emitted radiation, obtained as a Fourier transformation in the retarded time of the vector potential of Eq. (45). Assume that the magnetic field is that of an undulator with $N$ periods, so that the spectrum will consist of lines of width $1 / N$. Dropping the $y$ index, let us write the magnetic field as

$$
\Omega=\Omega_{0} \sin \omega_{0} t \quad ; \quad \omega_{0}=\frac{2 \pi c}{\lambda_{0}}
$$

with $\lambda_{0}$ the undulator period. In this field, an approximate expression for the instantaneous angle of the trajectory is

$$
\theta_{b}=\frac{k}{\gamma} \sin \omega_{0} t \quad ; \quad k=\frac{e}{2 \pi m c} \lambda_{0} B=\frac{\gamma}{c} \lambda_{0} \Omega_{0}
$$

The first Eq. (45) yields

$$
A_{p}(t)=\left(1-\gamma^{2} \theta_{b}^{2}\right) \Omega=\Omega_{0}\left(1-k^{2} \sin ^{2} \omega_{0} t\right) \sin \omega_{0} t
$$


showing that the spin independent radiation field only contains odd harmonics on axis (in this simplification, only 1 and 3).

The fourth Eq. (45) for the spin dependent radiation field reads

$$
A_{\sigma}^{(s)}(t)=-\left(1+\gamma^{2} \theta_{b}^{2}\right)\left[C_{1} \theta_{b} s_{x}+s_{z}\right] \Omega
$$

with the spin vector controlled by the BMT equation in the transverse field

$$
\frac{d \mathbf{s}}{d t}=C_{1} \mathbf{s} \times \Omega
$$

The vector equation (51) is equivalent to two second order scalar differential equations, formally identical for the two here considered components of the spin, of the type

$$
\frac{d^{2} s}{d t^{2}}-\left(\frac{1}{\Omega} \frac{d \Omega}{d t}\right) \frac{d s}{d t}+\Omega^{2} C_{1}^{2} s=0
$$

The exact integral of the above (Kamke $\# 2.79$, p.420) is

$$
\begin{gathered}
s(t)=-A \sin \left(b \cos \omega_{0} t\right)+B \sin \left(b \cos \omega_{0} t\right) \\
b=c_{1} \frac{\lambda_{0}}{2 \pi c} \Omega_{0}
\end{gathered}
$$

If we assume, as an example, that the beam is totally $x$ polarized at the entrance in the magnetic field, we have

$$
\left\{\begin{array}{l}
s_{x}=\frac{s_{x 0}}{\cos b} \cos \left(b \cos \omega_{0} t\right) \\
s_{z}=\frac{s_{z 0}}{\sin b} \sin \left(b \cos \omega_{0} t\right)
\end{array}\right.
$$

The functions in (54) cari be expressed using the generating series for the Bessel functions (Abramowitz-Stegun 7 p.361, 91:4.2, 9:1:4.3)

$$
e^{i z \sin \theta}=\sum_{k=-\infty}^{+\infty} J_{k}(z) e^{i k \theta}
$$


and the final expression for (50) reads

$$
\begin{gathered}
A_{\sigma}^{(s)}(t)=\Omega_{0}\left(1+k^{2} \sin ^{2} \omega_{0} t\right)\left[\begin{array}{c}
C_{1} H \sin ^{2} \omega_{0} t \sum_{0}^{\infty} \mathrm{J}_{2 \mathrm{n}+1}(\mathrm{~b}) \sin \left((2 n+1) \omega_{0} t\right) \\
+K \sin \omega_{0} t\left[\mathrm{~J}(b)+2 \sum_{0}^{\infty} \mathrm{J}(b) \cos \left(2 n \omega_{0} t\right)\right]
\end{array}\right] \\
H=\frac{4 k s_{x 0}}{\cos b} ; K=\frac{s_{20}}{\sin b}
\end{gathered}
$$

Both terms in the [..] of E.q.(56) contain odd harmonics, generated by the product of an even power of the sin function by a sin of an odd harmonic, or by an odd power of sin times a cos of an even harmonic.

A first competitor that can make this observation difficult is the finite emittance of the beam.

In this case, on axis, it is not $\Delta \phi=0$ but of the order of

$$
\Delta \phi \approx-\left\langle y^{\prime}\right\rangle \approx-\sqrt{\frac{\varepsilon_{y}}{\beta_{y}}}
$$

with $\left\langle y^{\prime}\right\rangle$ the average maximum vertical angle of the beam in the accelerator section containing the undulator. $\varepsilon_{y}$ and $\beta_{y}$ are the emittance and the $\beta$ twiss function. On axis, the function $A$ of Eq. (43) does not vanish, and we can wite, in place of the second of (45), the following

$$
A(t) \propto A \Omega=2 \gamma^{2} \theta_{b} \sqrt{\frac{\varepsilon_{y}}{\beta_{y}}} \Omega=2 \gamma \Omega_{0} \sqrt{\frac{\varepsilon_{y}}{\beta_{y}}} k \sin ^{2} \omega_{0} t
$$

The ratio between $A_{\sigma}^{\mathrm{s}}$ of Eq.(56) and $A_{\sigma}$ of Eq.(58) is

$$
\Re=\frac{2 \eta}{\gamma \sqrt{\varepsilon_{y} / \beta_{y}}}=g \frac{\hbar \omega}{2 m c^{2}} \sqrt{\frac{\beta_{y}}{\varepsilon_{y}}}
$$

This may impose a limit on beam emittance. It shows also that it is convenient to make the beam vertically parallel and wide (large $\beta_{y}$ ).

Fortunately, $A_{\sigma}$ contains only even harmonics also at higher order in the angles and $1 / \gamma$. Eqs. (56) and (58) show the same polarization, but do not appear in the same region of the spectrum.

Another effect that can compete to mask the spin dependent radiation in the odd harmonics and in the $\sigma$ polarization is due to undulator field imperfections. For instance, according to the second of 
Eqs. (44), a small $\delta \Omega_{\mathrm{x}}$ field residual component with the same periodicity of the main field will again produce an unwanted $A_{\sigma}$ on axis. A signal to noise ratio of the order of one is obtained when

$$
\frac{\delta \Omega_{x}}{\Omega_{y}} \leq \eta=\frac{g \gamma}{4} \frac{\hbar \omega}{m c^{2}}
$$

This has a value with the order of one for electrons and $10^{-5}$ for protons. It means that in order to measure the proton spin with this technique, the contribution to the field errors in phase with the field should be very small. A reasonable assumption (Brian Kincaid ${ }^{8}$, random walk argument) is that a random error $(\delta \Omega / \Omega)_{R}$ of the magnets that constitute the undulator will produce an upper limit of the error in phase, appearing in Eq. (60) of the order of

$$
\left(\frac{\delta \Omega}{\Omega}\right)_{\text {is phase }}=\frac{1}{\sqrt{N}}\left(\frac{\delta \Omega}{\Omega}\right)_{\text {random }}
$$

with $N$ the number of dipoles. One may think of many tricks to decrease the in-phase field error even further.

To complete this section, we should recall the expression for the intensity of undulator radiation in the fundamental line, in number of photons emitted per second and per unit horizontal and vertical angle, in radians 9

$$
\frac{d^{2} n}{d \theta d \phi}=\alpha N \gamma^{2} \frac{I}{e} F(k)
$$

where $I$ is the particle beam current and $F(k)$ a combination of Bessel functions.

\section{Compton ackscattering}

The radiation of a charged particle beam in an undulator shows a line spectra with a fundamental frequency located at

$$
\omega_{1}=\omega_{0} \frac{2 \gamma^{2}}{1+\frac{1}{2} k^{2}+\gamma^{2}\left(\Delta \theta^{2}+\Delta \phi^{2}\right)}
$$

and its harmonics. $\omega_{0}$ is defined in Eq. (47) and $\mathrm{k}$ is the deflection parameter defined in Eq. (48).

The frequency is the highest on axis. Spectrum lines have a width

$$
\frac{\delta \omega}{\omega}=\frac{1}{h N}
$$

with $h$ the frequency harmonic number and $\mathrm{N}$ the number of magnetic poles in the undulator. 
It is important to compare the radiation of an undulator with the radiation obtained by scattering of laser light by a charged particle beam. The scattered photon energy for head-on collisions, is given by

$$
\hbar \omega_{s} \cong \hbar \omega_{L} \frac{2(1+\beta) \gamma^{2}}{1+2(1+\beta) \gamma \frac{\hbar \omega_{L}}{m c^{2}}+\gamma^{2} \theta_{s}^{2}}
$$

where $\omega_{\mathrm{s}}$ and $\omega_{\mathrm{L}}$ are the frequency of the scattered and laser radiation, respectively, and $\theta_{\mathrm{S}}$ is the angle of the back scattered photon with resp it to the collision line.

Eq. (65) is very similar to (63). The main difference is the $1+\beta$ factor in the numerator that makes the up shifted frequency $\approx 4 \gamma^{2}$ times the original frequency instead that $2 \gamma^{2}$. This can be explained if one treats the radiation from an undulator as a scattering process of a photon and a charged particle (figure 4), first in the rest frame of the particle (PRF, p imed quantities) and then in the laboratory (LAB, unprimed quantities). In the particle rest frame the undulator is seen as an incoming e.m. wave, since relativity builds up an electric field next to the static field of the undulator.

It is in the $\mathrm{LAB}$ (undulator)

$$
\mathbf{E}=0 \quad\left\{\begin{array}{l}
B_{x}=B_{z}=0 \\
B_{y}=B_{0} \cos \omega_{0} t
\end{array} ; \quad \omega_{0}=\frac{2 \pi c}{\lambda_{0}}\right.
$$

and in the PRF

$$
\left\{\begin{array} { l l } 
{ E _ { x } = - \gamma \beta c B _ { 0 } \operatorname { c o s } \omega _ { 0 } t } \\
{ E _ { y } = E _ { z } ^ { \prime } = 0 }
\end{array} \quad \left\{\begin{array}{l}
B_{x}^{\prime}=0 \\
B^{\prime}{ }_{y}=\gamma B_{0} \cos \omega_{0} t \\
B^{\prime}{ }_{z}=0
\end{array}\right.\right.
$$

Note that this equivalent wave does not travel at the speed of light, but at the lower speed $\beta c$.

The undulator radiates. The $\mathrm{LAB}$ frequency of radiation is $\omega$. The $\mathrm{LAB}$ phase is

$$
\Phi=\omega(t-z / c)
$$

Time, space and frequency transform from LAB to PRF as follows

$$
t^{\prime}=\gamma_{2}\left(t-\frac{\beta_{z} z}{c}\right) \quad ; \quad z^{\prime}=\gamma_{z}\left(z-\beta_{2} c t\right) \quad ; \quad \omega^{\prime}=\gamma_{2} \omega_{0}
$$

and since the phase is an invariant 
(70)

$$
\omega(t-z / c)=\omega^{\prime}\left(t^{\prime}-z^{\prime} / c\right)
$$

combining Eqs.(65) and (66) and using the approximations in Eqs.(23) and (48), one obtains

$$
\omega=\omega_{0} \frac{1+\beta_{z}}{1-\beta_{z}^{2}} \cong \omega_{0} \frac{2 \gamma^{2}}{1+\frac{1}{2} k^{2}}
$$

that coincides, on axis, with Eq.(63).

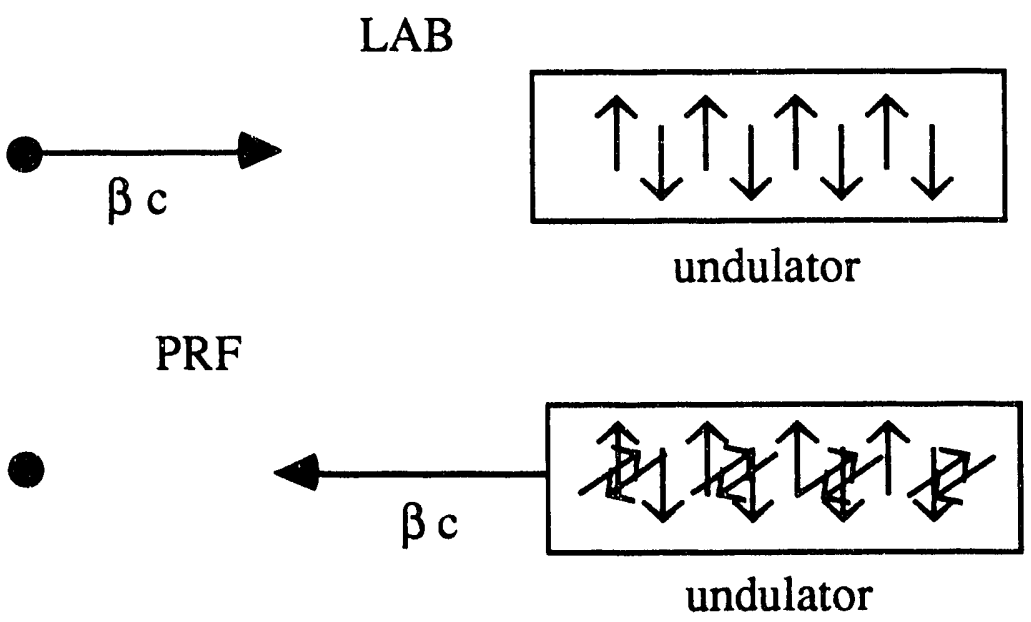

Fig. 4. The undulator as seen in the LAB and in the PRF

To study the kinematics of the scattering of laser photon by the charged particle beam, let us define the following quantities

$$
\begin{array}{ccc} 
& \text { energy } & \text { momentum } \\
\text { photon } & \varepsilon=\hbar \omega & \mathbf{k}=\frac{\hbar \omega}{c} \mathbf{n} \\
\text { particle } & E=\gamma m c^{2} & \mathbf{p}=\gamma m \mathbf{v}
\end{array}
$$

Compare the LAB with the PRF frames (figure 5).

In the $\mathrm{LAB}$, the conservation of momentum and energy is expressed by

$$
\left\{\begin{array}{c}
\mathbf{p}_{0}+\mathbf{k}_{0}=\mathbf{p}_{1}+\mathbf{k}_{1} \\
E_{0}+k_{0} c=E_{1}+k_{1} c
\end{array}\right.
$$

In the PRF, it is

$$
\left\{\begin{aligned}
E_{0}^{\prime} & =m c^{2} \\
\mathbf{k}_{0}^{\prime} & =\mathbf{p}^{\prime}{ }_{1}+\mathbf{k}^{\prime}{ }_{1} \\
E_{0}+k_{0}^{\prime} c & =E_{1}+k^{\prime}{ }_{1} c
\end{aligned}\right.
$$




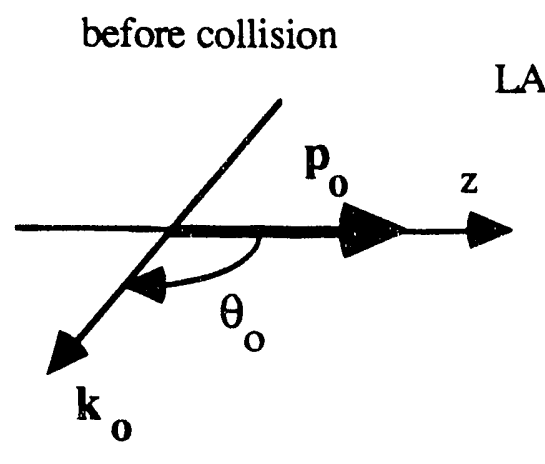

after collision

AB

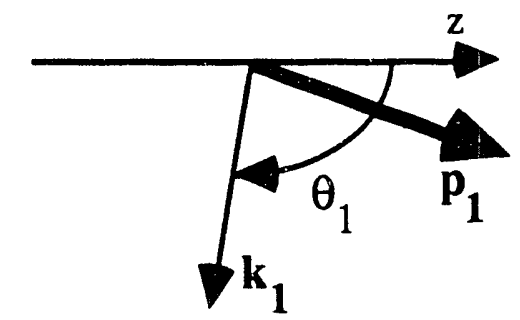

PRF

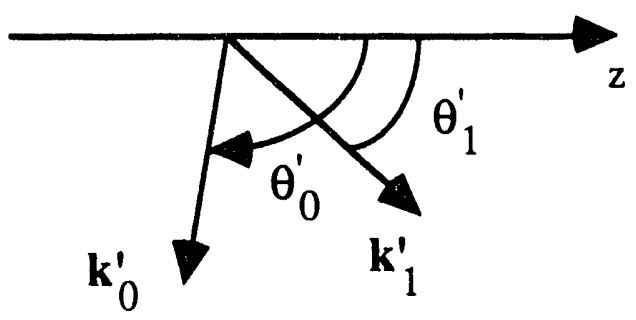

Fig. 5. Kinematics of Compton backscattering in the LAB and in the PRF

From Eqs. (74) the Compton scattering formula is derived in the PRF (primed quantities)

$$
k_{1}^{\prime}=\frac{k_{0}^{\prime}}{1+\frac{c k_{0}^{\prime}}{E_{0}}\left[1-\cos \left(\theta_{1}^{\prime}-\theta_{0}^{\prime}\right)\right]}
$$

Using the relativistic transformations of angles from PRF to LAB (unprimed quantities)

$$
\tan \theta=\frac{\sin \theta^{\prime}}{\gamma(\cos \theta+\beta)} \quad ; \quad \phi=\phi^{\prime}
$$

and assuming $\theta_{0}=\pi$ (backscattering), Eq. (65) is readily obtained in the LAB. Let us rewrite it in a more approximate form

$$
\hbar \omega_{s} \cong \hbar \omega_{L} \frac{4 \gamma^{2}}{1+\gamma^{2} \theta_{s}^{2}}
$$

Eq. (77) shows that the back scattered photon energy has a maximum forward, and decreases with the scattering angle. The radiation cone has a half-aperture defined by

$$
\gamma \theta \approx 1
$$


The intensity of the back scattered radiation is related to the luminosity of the process as

$$
n_{s}=\frac{N_{p} N_{L}}{\Sigma} f \sigma
$$

with $n_{\mathrm{s}}$ the number of back scattered photons per unit time, $N_{\mathrm{p}}$ and $N_{\mathrm{L}}$ the number of particles and primary photons contained in the volume of interaction, $\Sigma$ the common cross section of the particle and laser beams, $f$ the frequency of encounters, and $\sigma$ the scattering cross section.

If the particles have a spin, this contributes to the scattering cross section in Eq.(75), that can be written (Gustavson ${ }^{10}$ ) in the following way

$$
\sigma=\sigma_{0} \pm \sigma_{1} P_{L} P_{p} \cos \phi
$$

where $\sigma_{0}$ is the scattering cs for the unpolarized beam, $\sigma_{1}$ the spin dependent scattering cs, $P_{\mathrm{L}}$ the polarization of the laser (here assumed circular), $\mu_{\mathrm{p}}$ the particle spin (the earlier defined " $s "$ ) and $\phi$ the scattering angle relative to $P_{\mathrm{p}}$. Assume for the parameter appearing in Eq.(64)

$$
\xi=4 \gamma \frac{\hbar \omega_{L}}{m c^{2}} \ll 1 ; 1+\beta \approx 2
$$

then it can be shown that the unpolarized cross section is symmetric around the axis of scattering with a maximum there

$$
\left.\sigma_{0}\right|_{\max }=2 r_{0}^{2} \quad ; \quad r_{0}=\frac{\mu_{0}}{4 \pi} \frac{e^{2}}{m}
$$

with $r_{0}$ the classical radius of the particle, and that the polarized cross section is anti symmetric about the axis of scattering with maxima at angles determined by

$$
\gamma \theta_{s}=\frac{1}{\sqrt{3}}
$$

The ratio between maximum polarized cross section to maximum unpolarized is

$$
\frac{\left.\sigma_{1}\right|_{\max }}{\left.\sigma_{0}\right|_{\max }}=\frac{3 \sqrt{3}}{16} \xi=\frac{3 \sqrt{3}}{4} \gamma \frac{\hbar \omega_{L}}{m c^{2}}
$$

A comparison of the $\xi$ parameter of Eq. (81) for laser scattering with the $\eta$ parameter of Eq. (31) shows that the relative intensity of the polarized to unpolarized radiation is of the same order, as it should. 
As far as the absolute intensity is concerned, undulator radiation is proportional to the square of $\Omega$, Eqs.(4) and (8) and Compton backscattering to the square of $r_{0}$, Eq. (82), then inversely proportional to the mass of the particle.

In the scattering of virtual undulator photons, the number of primary photons to enter in Eq. (78) can be calculated by dividing the magnetic energy by the energy of a photon

$$
\frac{\varepsilon_{0} c^{2} B_{0}^{2}}{2 \hbar \omega_{0}} V
$$

with $V$ the volume of the particle beam bunch take takes part in the interaction.

\section{Conclusion}

A beam of charged particles with spin in relativistic motion can be probed by studying its radiation pattern in a static magnetic field (perhaps of an undulator). The pattern of synchrotron radiation will be used to image the beam on some optical detector, providing a precise and non invasive diagnostics ${ }^{11}$. The state of spin polarization in the beam can also be studied by synchrotron radiation or by Compton backscattering of laser light. The physical principles are similar for the two modes, with some important differences.

The undulator radiation shows the line structure of a diffraction pattern, with thinner lines the more the undulator periods are, and characteristic polarization properties. For practical cases, the frequencies lie in the infrared to soft $\mathrm{X}$-rays, with photon energies in the sub-eV to a few $\mathrm{KeV}$ range.

Compton backscattering produces a wide spectrum of radiation, correlated to the observation argle, with spectra from the $\mathrm{X}$-rays to $\gamma$-rays (photon energies ranging from a few $\mathrm{KeV}$ to hundreds of $\mathrm{MeV}$ ). If the undulator radiation is interpreted as a scattering of virtual photons, the expression for the luminosity shows that the number of undulator photons in the interaction region can be very large, since each photon (of frequency $\omega_{0}$ ) is much smaller than a laser photon (of frequency $\omega_{\mathrm{L}}$ ).

In either mode, the intensity of the observed radiation is inversely proportional to the square of the mass of the particle.

Tables 1 and 2 show the order of magnitude of some of the quantities in selected machines. We have considered two electron machines: the NSLS X-ray ring and APS and two proton machines: RHIC and SSC. 
Table 1. undulator and laser parameters

\begin{tabular}{|c|c|c|}
\hline & \multirow{4}{*}{$\begin{array}{c}\text { electrons } \\
0.511 \\
2 \\
7.9510^{-30}\end{array}$} & \multirow{4}{*}{$\begin{array}{c}\text { protons } \\
938 \\
5.58 \\
2.3610^{-36} \\
\end{array}$} \\
\hline$m c^{2}[\mathrm{MeV}]$ & & \\
\hline$g$ & & \\
\hline \multirow[t]{2}{*}{$r_{0}^{2}\left[\mathrm{~m}^{2}\right]$} & & \\
\hline & \multicolumn{2}{|c|}{ undulator } \\
\hline$\lambda_{0}[\mathrm{~m}]$ & 0.12 & 0.20 \\
\hline$B_{0}[\mathrm{~T}]$ & 0.4 & 3.2 \\
\hline$N$ & 25 & 20 \\
\hline$k=$ & $93.379 \lambda_{0} B_{0}=4.48$ & $0.05086 \lambda_{0} B_{0}=0.0326$ \\
\hline \multirow[t]{2}{*}{$\hbar \omega_{0}[\mathrm{eV}]$} & $2.0710^{-5}$ & $6.2010^{-6}$ \\
\hline & \multicolumn{2}{|c|}{ Laser scattering } \\
\hline$\hbar \omega_{L}[\mathrm{eV}]$ & \multicolumn{2}{|c|}{$\begin{array}{c}\mathrm{ND}-\mathrm{Yag}, 10 \mathrm{~mJ}, 1 \mathrm{KHz}, \lambda_{\mathrm{L}}=532 \mathrm{~nm} \\
2.33\end{array}$} \\
\hline
\end{tabular}


Table 2. selected colliders ${ }^{12}$

\begin{tabular}{|c|c|c|c|c|}
\hline & \multicolumn{2}{|c|}{ electron } & \multicolumn{2}{|c|}{ proton } \\
\hline & NSLS & APS & RHIC & SSC \\
\hline$\gamma(\approx \mathrm{GeV})$ & $4.910^{3} \quad(2.5)$ & $1.410^{4}(7)$ & $270(250)$ & $2.110^{4}(20 \mathrm{TV})$ \\
\hline $\begin{array}{c}\text { emittance } \\
{\left[10^{-9} \pi \mathrm{m}-\mathrm{rad}\right]^{\#}}\end{array}$ & 1 & $\begin{array}{l}7.4(\mathrm{H}) \\
0.77(\mathrm{~V})\end{array}$ & 9 & 0.05 \\
\hline current & $500 \mathrm{~mA}$ & $500 \mathrm{~mA}$ & $10^{11 / \text { bunch }}$ & $7.310^{9} /$ bunch \\
\hline beam radius [mm] & 0.10 & 0.16 & 5.4 & 0.026 \\
\hline \multirow[t]{2}{*}{ beta $[\mathrm{m}]$} & 10 & 10 & 100 & 100 \\
\hline & \multicolumn{4}{|c|}{ undulator radiation } \\
\hline$\hbar \omega_{1}[\mathrm{eV}]$ & 45 & 735 & 4.4 (5 $5^{\text {th }}$ harm $)$ & $5.610^{3}$ \\
\hline$\frac{a n}{d \theta d \phi}\left[1 / \mathrm{sec}-\mathrm{rad}^{2}\right]^{*}$ & $1.410^{24}$ & $2.410^{24}$ & $6.510^{22}$ & $4.110^{26}$ \\
\hline \multirow[t]{2}{*}{$\eta$, Eq. (31) } & 0.43 & $10(!)$ & $1.810^{-6}$ & 0.17 \\
\hline & \multicolumn{4}{|c|}{ backscattering } \\
\hline$\hbar \omega_{s}, \max$ & $220 \mathrm{MeV}$ & $1.8 \mathrm{GeV}$ & $66 \mathrm{MeV}$ & $4.2 \mathrm{GeV}$ \\
\hline$n_{\mathrm{S}}[1 / \mathrm{sec}]^{\$}$ & $2.110^{8}$ & $8.310^{7}$ & 0.030 & $7.210^{3}$ \\
\hline$\xi$, Eq. (81) & 0.089 & 0.26 & $2.610^{-6}$ & $2.110^{-4}$ \\
\hline
\end{tabular}




\section{References}

1B. W. Montague, Polarized Beams in High Energy Storage Rings Physics Reports 113 (1984) 1-90 J.Collins, S.F.Heppelman and R.W.Robinett (eds.) Polarized Collider Workshop Particles and Fields Series 42 American Institute of Physics, New York (1991)

${ }^{2}$ A. Luccio and S. Krinsky, in Physics of Quantum Electronics Vol. 8, Addison Wesley, Reading (1982) 181

${ }^{3} \mathrm{M}$. Conte and A. Luccio, Wiggler Snakes for RHIC Brookhaven Laboratory Accelerator Division AGS/ADD/Tech Note 366 (1992)

${ }^{4}$ A. A. Hahn and P. Hurh, Results from a Prototype Beam Monitor in the Tevatron Using Synchrotron Light Proc.. 1991 IEEE PAC, S.Francisco, May 6-9,1991, p.1177

5J. D. Jackson, Classical Electrodynamics Wiley, NewYork (1962), Chapters 11 and 14

${ }^{6} \mathrm{E}$. Kamke, Differentialgleichungen, Akademische Verlag, Leipzig (1967)

${ }^{7}$ M. Abramowitz, I. A. Stegun (eds.), Handbook of Mathematical Functions, Dover, New York (1986)

${ }^{8}$ B. M. Kincaid, J.Opt.Soc.Am., B2, (1985) 1294

9J. Kirz et Al. (eds.), X-ray Data Booklet Report Lawrence Berkeley Laboratory, PUB-490 (1985)

${ }^{10}$ D. B. Gustavson et Al., Nucl.Inst.Meth. 165, (1979) 177

M. Placidi, R. Rossmanith, Nucl.Inst.Meth., A214, (1989) 79

${ }^{11}$ A. Hofmann, Electron and Proton Beam Diagnostics with Synchrotron Radiation, Rept. CERN-ISR-TH/81-10 (1981), presented to the 1981 PAC

12 Review of Particle Properties, Phys.Rev., D45, 11 (1992) Part II, III-10 NSLS Design Book, Report BNL 6/6/78 Rev. 9/1/78

\# full energy

* no of photons/sec in the full first harmonic line, Eq. (62) in the text

$\$$ full spectrum, all angles, Eq. (79) in the text. It is assumed that the interaction length is $5 \mathrm{nsec}$ long. 

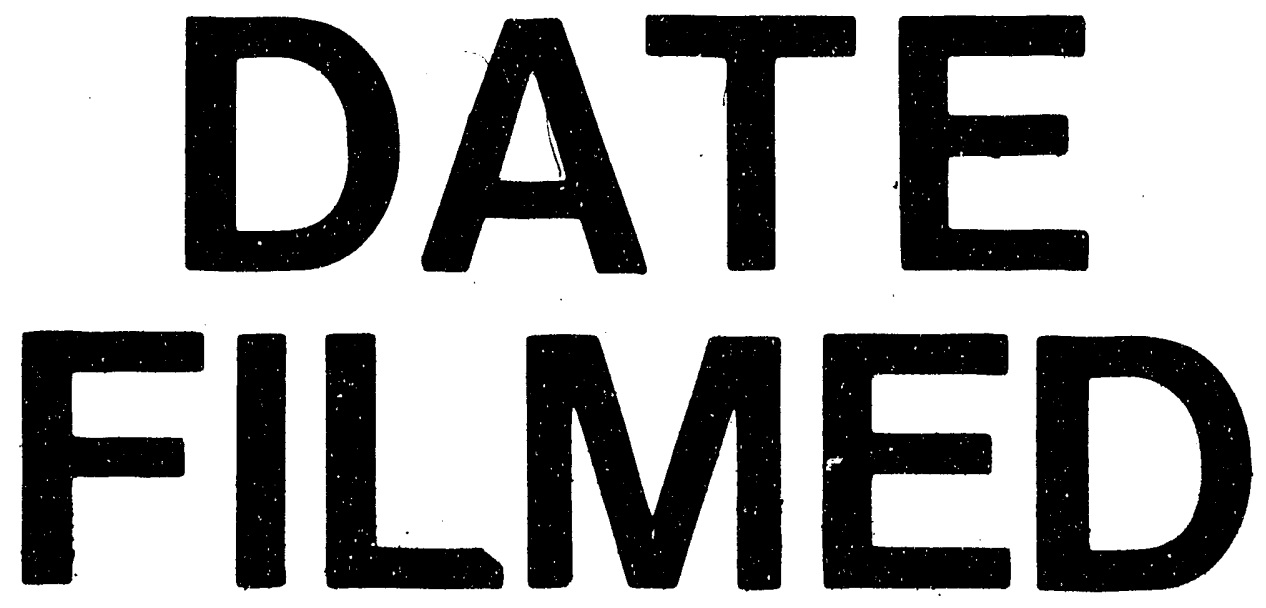

I

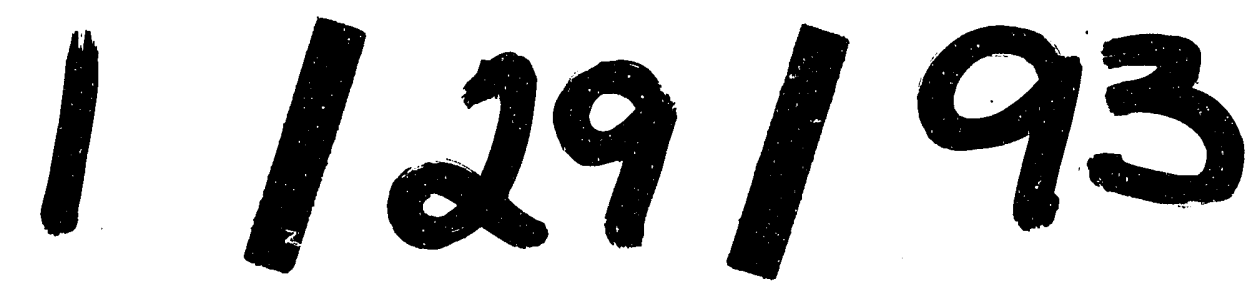


